\title{
Protection and Renewal of Historic Blocks Based on Architectural Typology
}

\author{
Hui Yu, Xinjie Sui, Mengfei Jiao \\ School of Architecture \& Fine Art, Dalian University of Technology, Dalian, China \\ Email:351311370@qq.com
}

How to cite this paper: Yu, H., Sui, X. J., \& Jiao, M. F. (2018). Protection and Renewal of Historic Blocks Based on Architectural Typology. Art and Design Review, 6, 115-124.

https://doi.org/10.4236/adr.2018.63011

Received: June 7, 2018

Accepted: July 14, 2018

Published: July 17, 2018

Copyright $\odot 2018$ by authors and Scientific Research Publishing Inc. This work is licensed under the Creative Commons Attribution International License (CC BY 4.0).

http://creativecommons.org/licenses/by/4.0/

\begin{abstract}
With the promotion and development of urbanization, historical blocks, with their historical value and particularity, have become the elements to avoid the homogenization of cities, and have been paid more and more attention by scholars in various fields. Taking three different types of historical blocks in Dalian (Nanshan District, Fengming Street, Dongguan Street) as examples, on the basis of typology, this paper compares different blocks, and puts forward such as repair prototype, integrate plane texture, remodel courtyard space and so on, in order to achieve the goal of protecting the historic district.
\end{abstract}

\section{Keywords}

Historic District, Extension and Reconstruction, Protection and Renewal

\section{Introduction}

With the development of urbanization rapidly, the phenomenon of urban homogeneity is becoming more and more serious, presenting the phenomenon of "thousands of cities are one side". The inheritance of history and traditional culture is facing a severe test. The historic district is an important cultural heritage of the city. It not only retains the local geographical features, but also reflects the life of the residents, arouses memories of local history, and finds their own sense of belonging. At present, although the importance of historic blocks has been recognized by most people, there are still a lot of problems in the process of specific protection. And many original distinctive neighborhoods gradually lose their own style. The construction of historic buildings and blocks of cities is irreversible. "Buildings in an area should vary in age and state and should include an appropriate proportion of old buildings. Old buildings are indispensable to the city, otherwise the development of streets and areas will lose vitality" (Jacobs, 
2006: p. 170). Once the building is gone, it will not be reappeared. Once the block is destroyed, it will be difficult to be repaired.

In fact, the continuity and reconstruction of the historic district mean to solve two problems: one is protection, another is development. Protection means to retain its own architectural style, inherit its historical culture and reconstruct the sense of place. Development is to constantly bring forth the new, meet the needs of social and economic development, and upgrade and change its original architectural function in the traditional cultural background. Therefore, how to balance the two relationships in the process of updating is the problem to be solved.

\section{Theoretical Basis of Research-Architectural Typology}

Italy architect Aldo Rossi was an important founder of typology. He summed up the experience of previous typology, joined his new rationalism, and provided the theoretical foundation of the narrow typology. He devoted himself to the internal structure of the city and architecture, and summarized its internal logic, and then reflected the form of the surface structure. His theory can be divided into two parts. The one is the typology based on rationalism and historicism, which is used to explain the relationship between the form and meaning of architecture, another is to emphasize the "collective memory" of places and cities, "The city is the place of collective memory, great ideas emerge from the history of the city, and shape the form of the city." (Rossi, 2006: pp. 130-131). Manfredo Tafuri, an Italian architectural theorist and architectural historian, regards typology as a critical thought, insists on the invariance of form. It is essentially a kind of urban criticism (Tafuri, 1980: pp: 158-159). Alan Colquhoun, an architectural theorist, believes that all thinking about architecture is limited to the attitude towards type, which constitutes that "the concrete practice of typology can convey artistic significance in society." (Colquhoun, 1991: pp. 247-248). Robert Kriel discusses the typology and morphology of the concept of urban space. He emphasized two important properties of urban space: the basic geometric configuration and the aesthetic effect of space. After collecting a large number of traditional urban space types, each type is defined, and the different forms are compared and analyzed (Kriel, 2007).

Typology essentially is to summarize and classify the forms with similar structural features, and then present the traditional images. History and culture are the collective memory of people in every region. No matter how the city develops, as long as it can touch the fragments of this kind of memory in the minds of the people, it will arouse the traditional thinking and present the memory of the tradition. The root of the study is to explore the deep structure of historical blocks that is the accumulation of social culture in the process of urban development-collective memory, and then reflecting it to the surface structure of the building form that is block, the organization and structure of the building. Applying typology to the conservation of historic blocks can provide an idea of ho- 
listic design. In this way, not only can it inherit the traditional style and features, raise the design depth, arouse the collective memory, but also constantly meet the material and cultural needs of the new life, and achieve the basic purpose of the protection of historic blocks.

\section{Research Method-Practical Application of Typology (Taking Historical Blocks of Three Spatial Types in Dalian as an Example)}

The research methods of typology can be simply summarized as follows: prototype discovery $\rightarrow$ simplification and reduction $\rightarrow$ refining prototype $\rightarrow$ redesign. Similarly, using typology in the historical block protection is first to investigate the status, access to data, draw charts to find, simplify, reduct and refine prototype, then to make plans, design operations, perform results, recreate and redesign. In this process, it presents tradition and changes the virtual existence of the mind into the existence of the entity. Analysis of specific types early can start from the following aspects: 1) The relationship between urban morphology and architectural group, including the relation between the general layout of the building group and the shape of the city; 2) Internal relations of building group, including building function and form, courtyard space form. By analyzing and extracting the architectural functions and forms adapted to different periods and styles of life, they can be used as the same prototype to carry out different types of transformations to keep the deep structure unchanged and form a variety of coordination.

\subsection{Enclosed Courtyard Block-Dongguan Street}

\subsubsection{Urban Morphology and Architectural Group Relationship}

Dongguan Street founded in the Japanese colonial period, is located in Dalian city Xigang Distric in Dalian. The middle of the block is divided into two sections by the city main road which is called the Yangtze River Road, covering an area of 7.64 hectares, including residential, commercial and market functions. Among them, residential building accounting for about $64 \%$, is the largest proportion. Other public facilities accounting for about $21 \%$, is scattered in the block. The whole block is flat, and remains the original surface texture in nearly a hundred years of historical accumulation (Figure 1, Figure 2).

\subsubsection{Building Group Internal Relations}

The spatial texture of Dongguan Street is different from the traditional Chinese "Li Fang" system and the modern arrangement. It was strongly influenced by the western city planning at that time, taking a strong form of organized groups. Each group is linked together whose shape is irregular quadrilateral, surrounding the contour line and conforming to the arrangement of the terrain in the base.

In historical blocks, the scale of the neighborhood is especially important. It 


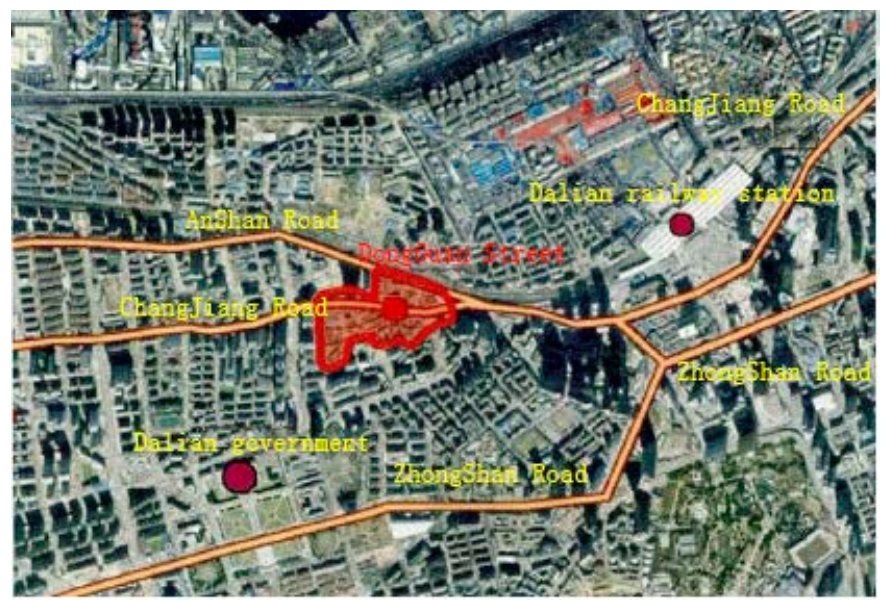

Figure 1. Dongguan Street District Bitmap.

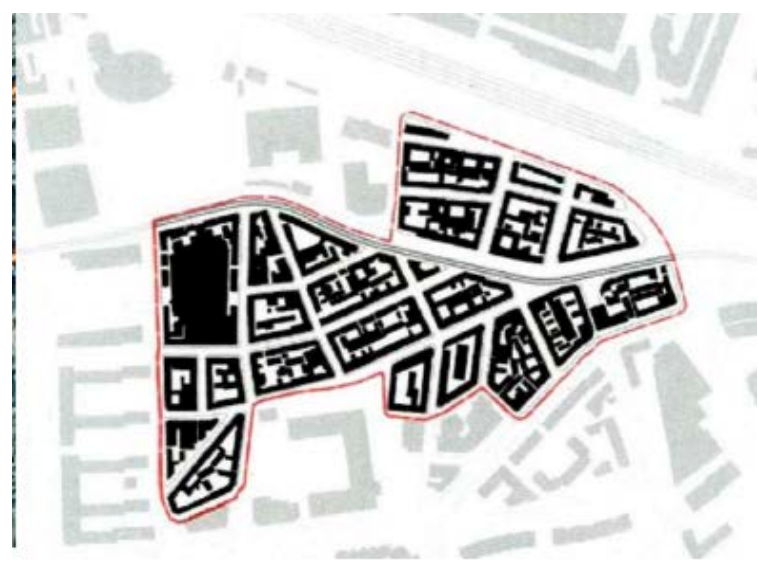

Figure 2. Dongguan Street Block Texture Map.

not only plays a role in transportation, but also serves as a link between different groups. As the city's living room with a centripetal force, each group will gather together, divided without dispersing. The narrow scale of Dongguan Street is more suitable for people to walk, creating a good business atmosphere with the neighborhood shops. It not only meets the traffic demands, but also gives the kind of comfort with people talking and shopping in the streets. But it is chaotic inside the street, inevitably causing the pressure to block traffic with the development of city. Similar to the structure of the early western block is Germany's Ritter Street (Figure 3). Facing many dilapidated old neighborhoods in Germany after World War II, German architects did not use bulldozers to demolish and rebuild all the blocks, but repaired and perfected the original block texture, protecting historical blocks, but not blindly following the traditional pattern of block structure. The original square structure is inserted into the “十” traffic space, thus changed into the “田” group layout (Figure 4). After darning, it not only meets the functional requirements of modern residential, and riches the block space, but also follows the original spatial texture blocks (Luan, 2014). 


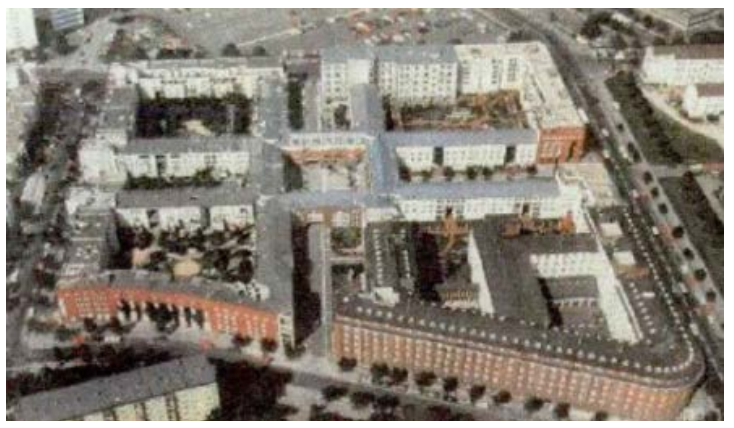

Figure 3. Germany's Ritter Street.

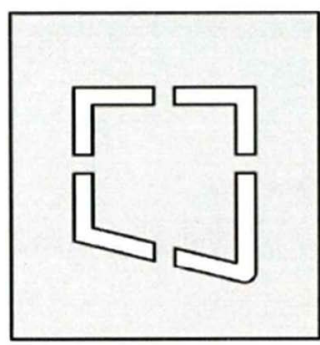

“口” layout

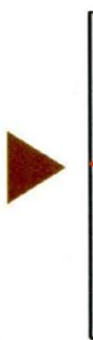

“十” weaving mesh

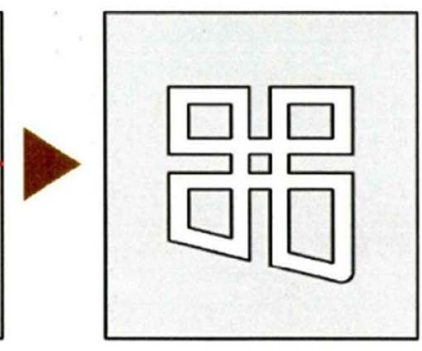

“田” layout

Figure 4. Analysis of Germany's Ritter Street.

\subsection{Bulk Block-Nanshan District Historic Block}

\subsubsection{Urban Morphology and Architectural Group Relationship}

Nanshan district historic block located at the foot of Nanshan mountain in Dalian, is part of the modern residential system with total area of 44 hectares and total construction area of 184,179 square meters. The south is near the hill and the north is near the lake (children's Park) with typical features of mountain architecture (Figure 5). There is of 30 meters and the slope difference of about $1 / 12$ to $1 / 11$ in terrain from north and south in the width of 350 meters. The road is basically arranged along the contour line, and the East-West elevation is also more than 10 meters in a height difference. As a result, the whole area is rich in spatial change. Because the road is arranged in accordance with the contour lines, so the block road is undulating with the ground, forming a network and visual momentum (Figure 6). The block was shaped basically around 1930s by the Japanese. Most of them referring to the nordic style of architecture are different styles of western style villa with whole Japanese architecture composition. In order to adapt to the climate of Dalian, the Japanese designers first use the wall brick, giving higher the value of building research to Nanshan historic district.

\subsubsection{Building Group Internal Relations}

The form of historical blocks in Nanshan District is scattered block, there being no obvious group and formal division, scattering on the base along the terrain with the full of change of courtyard space. Because of the terrain, there are differences in height from the road to the courtyard with several steps. So standing 


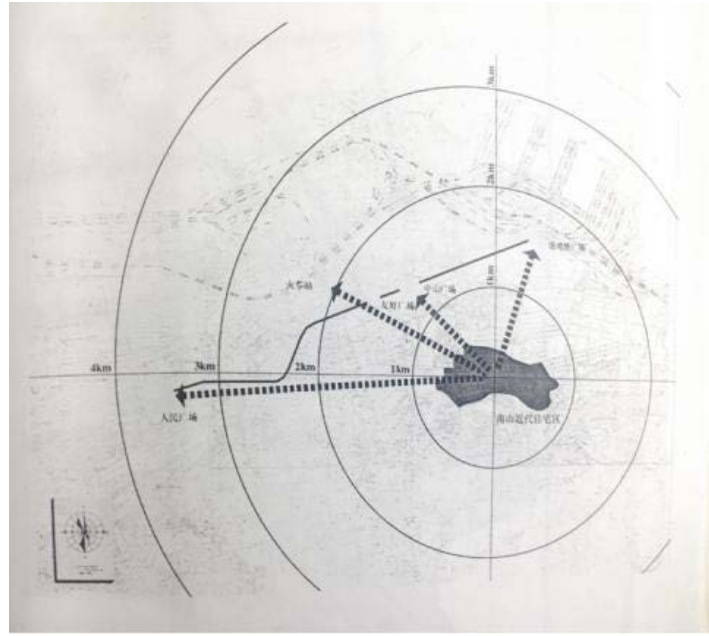

Figure 5. Nanshan Block District Bitmap.

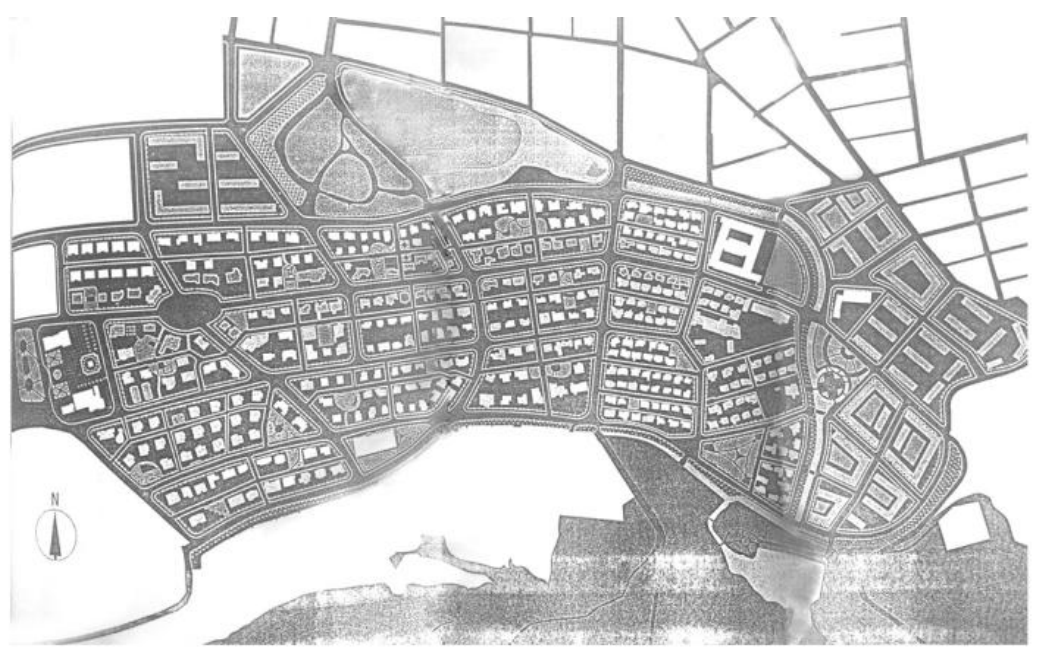

Figure 6. Nanshan Block Texture Map.

in different parts of the block, there will be different spatial feelings. The design of the building itself is combined with the elevation difference, setting two entrances and exits, the south exit to the courtyard space, the north entrance to the garage and so on. The use of the height difference is very natural and ingenious.

One part of Nanshan historic courtyard is basically composed of building walls, and other factors, another composed of subgrade construction and falling, and others are from new buildings and structures, or plant composition. There are big and small in size, varies in space form and quality. Because the block has undergone great changes in the people living here with economic development, improvement of living standards, changes in lifestyle, and the needs of space environment since a hundred years, leading that some simple function of the original residence has been unable to meet modern people's life. So the whole block has the following problems:

1) The phenomenon of reconstruction and construction is very serious. Although it meets some temporary requirements of the residents, it has a very bad 
influence on the overall style and landscape of the historical district of Nanshan, leading to a larger building density and a cluttered architectural style.

2) The design of courtyard wall is too single, for example, the lack of lower walsl, fences of good vision or other retaining structure. Both landscape and psychology require a more open space. Therefore, combined with the life safety and public buildings and other needs, opening the walls appropriately is conducive to the whole area of open space. Not only can it meet the psychological needs of modern people, but also enhance people for architectural appreciation.

3) The architectural nature of many houses has changed. It turned out that the buildings which only used as residential functions are now changed into commercial functional buildings. The change of function will inevitably lead to changes in the spatial structure of the blocks, so the neighborhood is losing its former style.

\subsection{Chain Street Blocks-Fengming Street Block}

\subsubsection{Urban Morphology and Architectural Group Relationship}

Fengming street block is located in the center of Dalian, covering an area of about 124,800 square meters, a total length of about $1200 \mathrm{~m}$ from the west to the East with zonal distribution. Modern Japanese residential buildings arranged on both sides of Fengming street which is as main axis (Figure 7). The similar to Nanshan historic district, Fengming street is also designed by Japanese architect that returned from Europe and America. Because of the impact by the western modernism design idea, they try to combine with the western European style garden and Eastern architecture, thus forming a new architectural style.

\subsubsection{Building Group Internal Relations}

Fengming street is in the form of a chain, the whole block is separated and also closed, both be linked and unlocked. The block is rectangle divided into nine plots which are distributed in the two sides of FengMing street which is viewed as central axis. The size of each block is similar, arranged in the form of a linear grid. Courtyard space is rich, there being a clear level of space, from the "inter room" space through its own transformation into "house" space, "home" space formed by the housing space and wall combined, the courtyard space formed by the combination of the "home" space, the block space formed between the courtyard space combined, Finally, the block spaces that are combined to form the whole district space. That is, "inter room" $\rightarrow$ "house" space $\rightarrow$ "home" space $\rightarrow$ "courtyard" space $\rightarrow$ "block" space $\rightarrow$ "district" space in the form of hierarchy (Wang, 2014) (Figure 8).

\section{Specific Protection Methods of Continuation and Reconstruction}

\subsection{Integrate Plane Texture-Enclosed Courtyard Blocks (Dongguan Street)}

Through the analysis of three aspects of the Dongguan Street above, it can be 


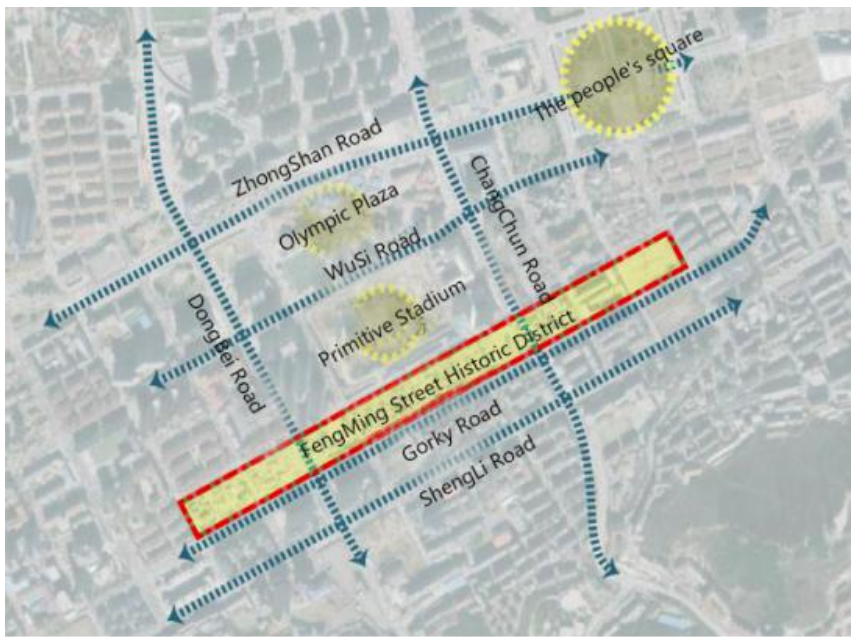

Figure 7. Fengming Street District Bitmap.

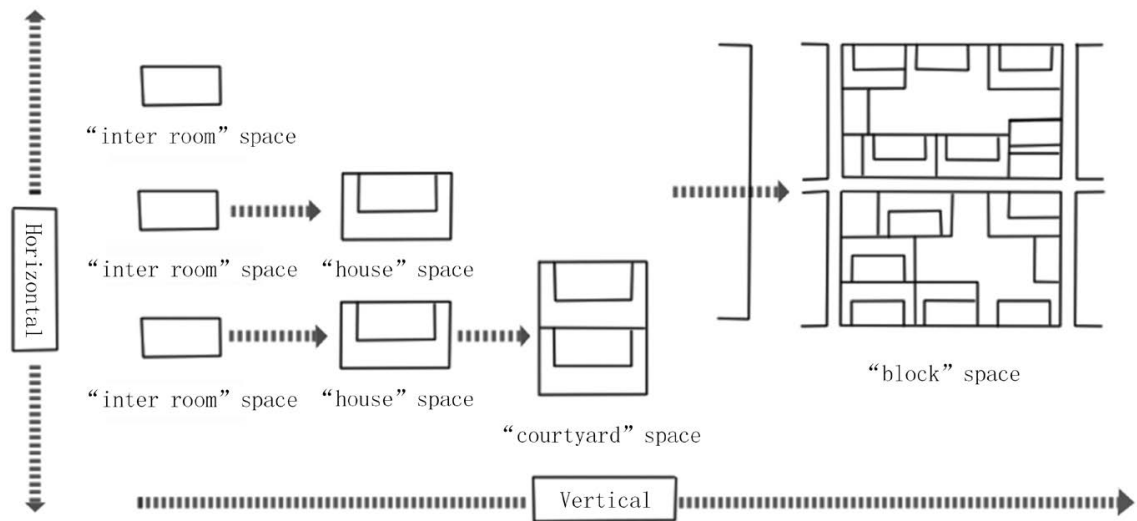

Figure 8. Analysis of space formation of Fengming Street.

drawn that the function of Dongguan Street buildings is complex, distribution is scattered, city form docking is not perfect, and the internal block road is chaotic. The typology method introduced is for highlighting the importance of the functions in this historic development, making its development in the future to continue to improve, achieving the sustainable development of the city. Using the method of the mending of the plane texture will integrate and classify the same functionality of architectural group, regularing the road of confused blocks, and taking the road as the skeleton to increase, reduce or change the local geometry blocks to re programming blocks.

Concrete operation:

1) Referring to the texture and structure of the urban spatial pattern, on the premise of satisfying the road traffic function of the block to organize the texture of block and improve the graph base relationship between the block and the urban texture; 2) According to the division, it is necessary to organize the blocks in the form of function units and plan the block roads.

3) According to the principle of mend and integrate, combing network and partition, deleting the construction appropriately, reducing the volume of con- 
struction, increasing the rate of greening and road blocks is for regularity.

\subsection{Remodel Courtyard Space-Bulk Block (Nanshan Historic Block)}

The analysis of Nanshan historical block shows that the courtyard has become a more characteristic place, but there are many problems in the bulk block. First of all, architectural arrangement is irregular whose layout is scattered, leading to the dock of space texture and the city is not very good. Secondly, the courtyard piled up debris and a lot of new buildings built lead that the building density is too large and the whole block because of the excessive period of architecture and style is not uniform. Again, some walls being too big affect to appreciate the building from the street. Moreover, the enclosure elements of the courtyard are relatively single and unrich. Finally, the level of the space from the street to the courtyard is somewhat too direct and rough. Using the typology principle for the entire building blocks to do a statistical analysis is to extract the architectural elements of traditional symbols of unity and apply it to repair the old buildings so that the whole block to achieve the purpose of harmony and unity.

Concrete operation:

1) Sorting out the relationship between the first layer of texture, and making a preliminary plan for the block are to achieve the maximum degree of integration.

2) To dispose the second layers of texture, reducing the density of overall block building, evacuating the distance between buildings, perfecting the arrangement and combination methods by removing redundant buildings and affecting the overall planning of buildings are to realize the integration of block buildings.

3) The extracted traditional architectural symbols are used on the facade of the building to renovate the old buildings and to change the style of the new buildings.

4) Redesigning some walls, reducing the height of walls combined with greening, strengthening courtyard elements, considering some wooden fence, wooden pillars, low wall enclosurein in addition to the entity of the wall are to enhance the visual effect.

5) Strengthening the level of space and transition, the level from the public space $\rightarrow$ semi private and semi public space $\rightarrow$ private space should be rich. Some public buildings for around can be fully open, arranging some wooden fence, low wall or flower beds to transit space symbolically.

6) The design of the courtyard should be combined with the natural height difference to form a rich variety of space. There are also many kinds of forms, such as the front yard and the back garden (Shao, 1999).

\subsection{Repair Prototype-Chain Street Blocks (Fengming Street)}

Through the analysis we can conclude that Fengming Street with its unique order in space structure, clear divisional hierarchy and regular and compact nine 
plots, is a typical representative of the old block texture space and need to be preserved and inherited. However, in the past hundred years of historical accumulation, the architectural style has been greatly damaged, and many of the old buildings have lost their original shape, that only is distinguished vaguely by the outline after wind broken, causing great losses for the whole city culture.

Concrete operation:

For the historic district of reasonable space order and architectural style uniform, the method of the most economical and reasonable is the prototype repair. According to the external form of the existing building, relying on computer aided modeling design is to restore the original style of the building, and adopt new materials and new technologies to restore the historical building. Not only can it retain the traditional elements, but also meet the needs of modern living, inherit historical culture, and achieve sustainable development.

\section{Conclusion}

In this paper, three historic blocks in Dalian are taken as examples. Methods of block protection for different spatial types are proposed based on typology theory. The typology applied to the historical street protection is of great significance, not only to inherit the historical architectural elements, preserve the historic buildings, stimulate memories of historical context in the new context, but also to meet people for the new lifestyle needs. In a word, historical blocks play an important role in the process of urban development, being the epitome of a city's history, ideas and culture, place spirit with great research value. History will not reproduce, but through the real physical protection of historic buildings is handed down from age to age. It's the responsibility of every architect's bounden.

\section{References}

Colquhoun, A. (1991). Modernity and Classical Tradition: Architectural Essays 1980-1987 (pp. 247-248). Cambridge: MIT Press.

Jacobs, J. (2006). The Death and Life of Great American Cities (p. 170). Trans. Hengshan Jin. Nanjing: Yilin Press.

Kriel, R. (2007). Town Spaces: Contemporary Interpretations of Traditional Urbanism. Trans. Qiuye Jin. Beijing: China Building Industry Press.

Luan, Y. F. (2014). Research on the Protection and Renewal of Urban Historical Blocks. Dalian: Dalian University of Technology.

Rossi, A. (2006). The Architecture of the City (pp. 130-131). Trans. Shijun Huang. BeiJing: China Building Industry Press.

Shao, M. (1999). Research on Planning and Design of Modern Residential Area in Nanshan of Dalian City. Dalian: Dalian University of Technology.

Tafuri, M. (1980). Theories and History of Architecture (pp. 158-159). Trans. Giorgi Verrecchia. New York: Happer \& Row.

Wang, Z. (2014). An Analysis of the Spatial Structure of the Historical District of Fengming Street in Dalian. Dalian: Dalian University of Technology. 